\title{
On the Existence of Solar-type Activity in the Secondary Components of Cataclysmic Variables
}

\author{
E.S. Dmitrienko \\ Crimean Astrophysical Observatory, Nauchny, SU-334413, Crimea, USSR
}

\begin{abstract}
Cyclic variations of the parameter $O-C$ (the difference between observed and calculated times of the light minimum in eclipse) have been detected for Nova 1934 DQ Her in the $U B V R I$-bands during 1982-89 with a recurrence time of about 5-6 years and amplitudes of about 2-4 min. No $O-C$ oscillations for the nova-like $\mathrm{AC}$ Cnc have been discovered with the resolution of $45 \mathrm{sec}$ in 1985-89. Both systems consist of a white dwarf and a late-type dwarf component. Some possibilities to explain their $O$ - $C$-behaviour are discussed.
\end{abstract}

Cyclic variations of $O-C$, observed as inherent for different types of cataclysmic objects, such as polars, post novae, nova-like, dwarf novae, allow us to propose that the physical nature of this phenomenon could be uniform for these binaries. Several hypothesis are known today for the explanation of the cyclic $\mathrm{O}-\mathrm{C}$ variations in binary systems. There are, for example, apsidal motion or the existence of a third body (Stern, 1939), eccentricity of the accretion disc around the white dwarf (WD), solar-type magnetic activity of secondaries (Bianchini, 1988; Applegate and Patterson, 1987; Warner, 1988) and swinging of WD-magnetic axis (Andronov, 1987). To choose between these hypothesis, some general properties of cataclysmic variables (CV) can be mentioned. Gas streams from the secondary form an accretion disc around a WD if the magnetic field of the latter is smaller than $10^{7}-10^{8} \mathrm{G}$. In polars there are no accretion discs. So the hypothesis of an eccentric disc as a common cause of cyclical variations of $O-C$ in all $\mathrm{CVs}$ is not applicable. The existence of the apsidal motion can be tested by observing systems with eclipses of both components. This motion produces oscillations of $O-C$ values in such a way that the primary and secondary minima are displaced with respect to each other. When a third body is present, the time-dependence of $O-C$ of both minima shows oscillations only. However, in CVs the $U B V R I$-luminosity of the secondaries is so small that we can observe both minima in systems with $P>6^{d}$ only (Patterson, 1984). No published light curves of these CVs are available and hence we have observed in 1985-1989 the eclipsing nova-like system AC Cnc, which has a period $7^{h} 12^{m}$. We also examined the time-dependence of $O-C$ in Nova 1934 DQHer in 1982-1989, with period $4^{h} 39^{m}$. 
The UBVRI-light curves of DQHer and ACCnc, with almost complete orbital phase coverage, have been obtained in Crimean Astrophysical Observatory using the 1.25-meter reflector. The photometer-polarimeter of Helsinki University Observatory (Piirola, 1973) was used. The observational technique is described by Dmitrienko et al. (1985). Here we give only the results of the $O-C$ observations.

The value of $O-C$ of $\mathrm{DQHer}$ is decreasing with the rate $\Delta(O-C) / \Delta t=10^{-6}$ in 1982-1989 and has cyclic variations with recurrence time of about 5-6 years and amplitude about 2-4 min (Fig. 1). No $O-C$ oscillations for $\mathrm{AC} \mathrm{Cnc} \mathrm{have} \mathrm{been}$ detected with a resolution of $45 \mathrm{sec}$ in 1985-1989 for either minimum. According to our observations, there is no eccentricity in accretion disc, no apsidal motions and no third body in AC Cnc. The differing behaviours of the observed parameter $O-C$ in AC Cnc and DQ Her may indicate 1) that if there is cyclic magnetic activity of the secondary in $\mathrm{CV}$, the effect of this activity on the value of $O-C$ can be detected only in the systems with $\left.P<7^{h} ; 2\right)$ that there exists some intrinsic difference between secondaries of cataclysmic systems with periods less and greater than 7 hours.

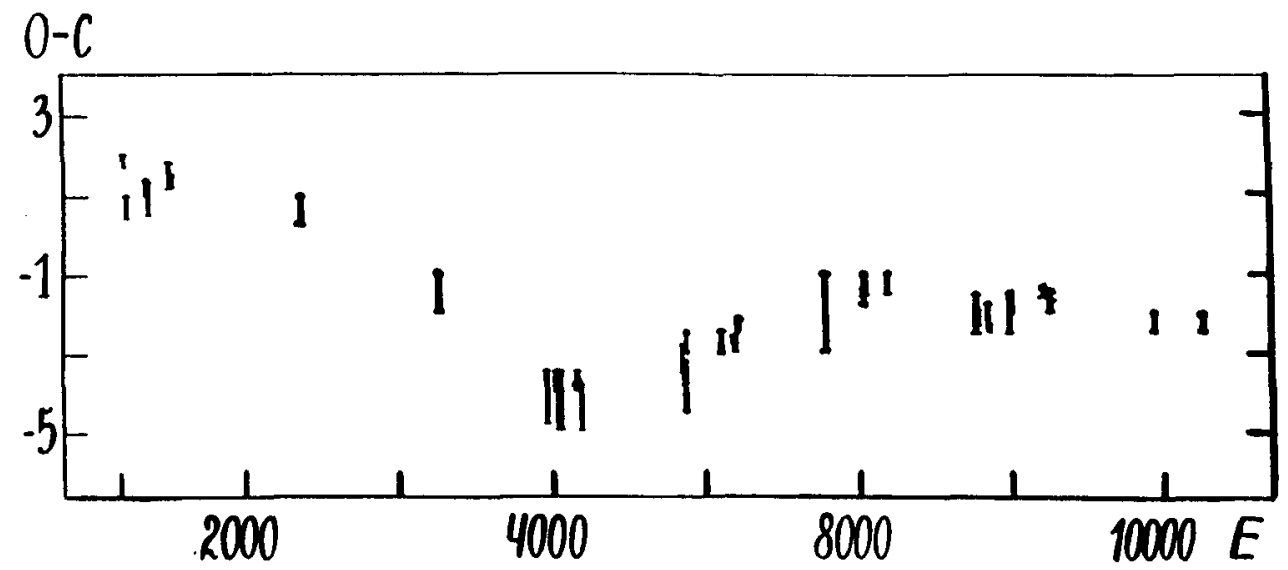

Fig. 1. The time dependence of the $O-C$ for DQ Her in 1982-89. The units of $O-C$ are minutes, and the units of the time the number of the double value of the orbital period. The latter is $4^{\mathrm{h}} 39^{\mathrm{m}}$.

\section{References}

Andronov, I.L.: 1987, Astrophys. Space Sci. 131, 557

Applegate, J.H. and Patterson, J.: 1987, Astrophys. J. 332, L99.

Bianchini, A.: 1988, Inf. Bull. Var. Star no 3136, 1

Dmitrienko, E.S., Efimov, Y.S., Shahovskoy, N.M.: 1985, Astrofizika 22, 31

Patterson, J.: 1984, Astrophys. J. Suppl. Ser. 54, 443

Piirola, V.: 1973, Astron. Astrophys. 27, 383

Stern, T.E.: 1939, Mon. Not. Royal Astron. Soc. 89, 451

Warner, B.: 1988, Nature 336, 129 